\title{
INSIDE THE CENTRAL CAVITY
}

\author{
PETER G. MEZGER \\ Max-Planck-Institut für Radioastronomie \\ Auf dem Hügel 69, 53121 Bonn, Germany \\ AND \\ SABINE PHILIPP \\ Max-Planck-Institut für Radioastronomie \\ Auf dem Hügel 69, 53121 Bonn, Germany \\ Institut für Theoretische Astrophysik \\ Tiergartenstrasse 15, 69121 Heidelberg, Germany
}

The Circum-Nuclear Disk (CND) is probably a by-product of the explosive event which created Sgr A East. Its inner edge of radius $\mathrm{R} \sim 1.7 \mathrm{pc}$ is well developed and comprises the Central Cavity (CC) which is filled with $\sim 260 \mathrm{M}_{\odot}$ of ionized gas. ISM appears to flow through the CND into the Central Cavity at a rate of $\dot{M} \sim 0.01 \mathrm{M}_{\odot} \mathrm{yr}^{-1}$. Dust and Lyman continuum luminosities of the central 1.25 parsec, corrected for photons not absorbed by dust and gas inside the CC are $\sim 10^{8} \mathrm{~L}_{\odot}$ and $\sim 10^{51}$ Lyc photons s${ }^{-1}$, most of which are provided by a cluster of 24 hot and massive stars, which also provide $\sim \frac{1}{3}$ of the integrated K-band flux density (see the recent review by Mezger et al., 1996). A larger number of cool M- and K-giants and more than a million low-mass MS stars provide the remaining flux density, which comes from a diffuse background emission first seen in our NIR mosaic maps (Zylka et al., 1997).

The compact synchrotron source Sgr $\mathrm{A}^{*}$ with a mass of $\sim 2-3 \times 10^{6} \mathrm{M}_{\odot}$ appears to be a starving black hole. It has a well developed radio spectrum but only upper limits for the flux densities in the NIR and X-Ray regimes. The luminosity of $\mathrm{Sgr}^{*}$ is only a few hundres $\mathrm{L}_{\odot}$. The true size of the radio source appears to have been resolved by mm-VLBI (Duschl, 1997).

The spectrum integrated over the central parsec is dominated by stellar radiation reprocessed by dust and resembles the spectrum of a Seyfert 2 galaxy. The reprocessed radiation masks completely the radiation associated with $\mathrm{Sgr} \mathrm{A}^{*}$. More details, related specifically to the stellar population within the central $1.25 \mathrm{pc}$ will be presented in a forthcoming paper (Philipp et al., 1997). 


\section{References}

Duschl W.J., 1997, in these proceedings.

Mezger P.G., Duschl W.J., Zylka R., 1996, A\&AR, 7, 4.

Philipp S., Zylka R., Mezger P.G. et al., 1997, in prep.

Zylka R. et al., 1997, in these proceedings. 